\title{
Harnessing Surface-Functionalized Metal-Organic Frameworks for Selective Tumor Cell Capture
}

Xiaoyue Qi, ${ }^{\dagger}$ Ziyong Chang, ${ }^{\ddagger}$ Duo Zhang, ${ }^{\S}$ Kellie J. Binder,, Sensen Shen, ${ }^{\dagger}$ Yan Yan Shery Huang, ${ }^{\S}$ Yu Bai, ${ }^{\dagger}$ A Andrew E. H. Wheatley, ${ }^{*}, \| \odot$ and Huwei Liu ${ }^{*}+{ }^{\dagger}$

${ }^{\dagger}$ Beijing National Laboratory for Molecular Sciences, Key Laboratory of Bioorganic Chemistry and Molecular Engineering of Ministry of Education, Institute of Analytical Chemistry, College of Chemistry and Molecular Engineering, Peking University, Beijing 100871, China

${ }^{\ddagger}$ School of Chemical Engineering, The University of Queensland, St. Lucia, Brisbane, Queensland 4072, Australia

${ }^{\S}$ Department of Engineering, University of Cambridge, Trumpington Street, Cambridge CB2 1PZ, United Kingdom

"Department of Chemistry, University of Cambridge, Lensfield Road, Cambridge CB2 1EW, United Kingdom

\section{Supporting Information}

$\mathrm{M}$ etal-organic frameworks (MOFs) have risen to prominence as organic-inorganic hybrid materials with porous structures. ${ }^{1}$ MOFs externally modified using different functionalities have emerged as the basis for a new breed of functional material that offers an array of applications in drug delivery, sensing, biocatalysts, and cell imaging. ${ }^{2,3}$ Of course, if they are loaded with therapeutic molecules, MOFs have been shown to be capable of efficient drug delivery. ${ }^{4,5}$ This means that the possibility of combining functional MOFs with the carrying of payloads suggests a general synthetic strategy by which to prepare antibody-functionalized materials to achieve both targeted cell capture and drug delivery.

Tumor cell analysis plays a vitally important role in cancer diagnosis, drug screening, and clinical therapeutics. ${ }^{6,7}$ Circulating tumor cells (CTCs) are capable of initiating cancer metastasis by migrating through the vascular system, ${ }^{8}$ with the potential to reflect the molecular profile and developmental stage of the primary tumor they escaped. ${ }^{9}$ This renders their analysis vital to understanding both the mechanism of tumor metastasis and offering a prognosis of the primary cancer. However, the rarity of CTCs makes their detection and analysis a significant challenge; typically only hundreds of CTCs coexist with $10^{9}$ hematologic cells. ${ }^{10}$ It has also been shown that CTCs are significantly heterogeneous, complicating recognition. ${ }^{11}$ The necessity of capturing CTCs selectively and efficiently has led to the development of a variety of functional materials and devices. ${ }^{12,13}$ However, for applications in anticancer drug screening, it is necessary to fabricate multifunctional platforms that combine CTC recognition and capture with controlled drug release.

Here, we successfully fabricate a platform for selective CTC sequestering that also has the potential for targeted drug delivery and screening using a composite chip with integrated dual functionality. Zn-based MOFs (ZnMOFs) have exhibited diverse practicality with applications reported in the fields of sensing, ${ }^{14}$ imaging, ${ }^{14}$ and catalysis. ${ }^{15}$ The fabrication of a rare example of a $\mathrm{ZnMOF}$ bearing free carboxylic acid groups (ZnMOF-COOH) offers a flexible and scalable platform for the creation of peptide linkages. This in turn offers a general method to conjugate large molecules, such as enzymes and antibodies, to the MOF and provides a simple route to a highly effective immunotrapper. Hence, the free acid groups borne by the MOF have undergone postsynthetic modification (PSM) to express antiepithelial cell adhesion molecule (anti-EpCAM) antibody (see Supporting Information). EpCAM itself is a transmembrane glycoprotein expressed exclusively in epithelia and epithelial-derived neoplasms. The up-regulation of EpCAM (100-1000-fold) relates to cancer progression in virtually all epithelial carcinomas, making it a promising candidate for immunotrapping strategies. ${ }^{16}$ The synthetic strategy by which PSM gives an immunotrapper functionalized by anti-EpCAM is summarized in Scheme 1.

In this work, immunotrapper development involved fabricating a $\mathrm{ZnO}$ substrate via high-temperature sintering, on which was grown a Zn-based MOF (see Supporting Information for full details of immunotrapper preparation and characterization). Scanning electron microscopy (SEM) of the $\mathrm{ZnO}$ substrate revealed a nanostructured morphology

Scheme 1. Schematic Illustrating the Synthetic Strategy for Fabricating a MOF-Based Immunotrapper

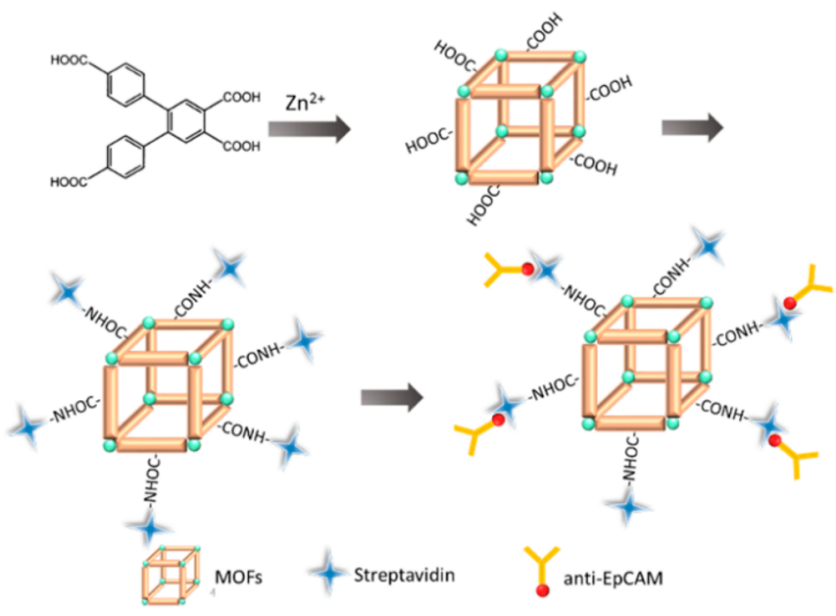

Received: August 2, 2017

Revised: September 25, 2017

Published: September 25, 2017 
(Supporting Information Figure S1). Nano-ZnO substrate was next added to a Teflon lined stainless-steel autoclave together with $\mathrm{Zn}\left(\mathrm{NO}_{3}\right)_{2} \cdot 6 \mathrm{H}_{2} \mathrm{O}, 1,1^{\prime}: 2^{\prime}, 1^{\prime \prime}$-terphenyl-4,4',4",5' -tetracarboxylic acid $\left(\mathrm{H}_{4} \mathrm{ttca}\right)$ and $4,4^{\prime}$-bipyridine before being heated to $160{ }^{\circ} \mathrm{C}$ for $120 \mathrm{~h}$. X-ray diffraction suggested the resulting material to be a composite in which nano- $\mathrm{ZnO}$ was coated by ZnMOF-COOH (Figure S2). ${ }^{17}$ The latter was characterized by $\mathrm{N}_{2}$ gas sorption at $77 \mathrm{~K}$ (Figure S3), revealing a surface area of $22.9 \mathrm{~m}^{2} / \mathrm{g}$ and a pore diameter of $158.2 \AA$. These data are as expected. ${ }^{17}$ The successful synthesis of ZnMOF-COOH, including the presence of uncoordinated carboxylic groups, was further confirmed by Fourier transform infrared (FTIR) spectroscopy (Figure S4). ${ }^{18}$

Remarkably little work has been done on derivatizing MOFs that bear free acid groups. An organic amine catalyst has been hosted by a MOF through acid-base interactions ${ }^{19}$ while simple esterification has recently been reported. ${ }^{20}$ However, we reasoned that PSM should enable the creation of peptide linkages on MOFs in a way that promises the wide-ranging ability to covalently immobilize biomacromolecules. ${ }^{21,22}$ This would represent a significant step, with extant research pointing to the covalent immobilization of antibodies offering significant advantages over their passive adsorption on substrates. Benefits would include enhanced stability, dispersibility in the aqueous phase, control of antibody surface density, improved structure retention, and reduced blocking of the antigen binding site. $^{21,23,24}$ Furthermore, immobilization of enzymes on solid supports can enhance stability and proteolysis efficiency, as well as ease of separation and recovery for reuse. ${ }^{25}$ In this work, we validate our thesis by modifying the surface acid groups on $\mathrm{ZnMOF}-\mathrm{COOH}$, thus conjugating the biotin-sequestering protein streptavidin (SA) to the $\mathrm{ZnMOF-COOH}$ via incubation in the presence of the activators $N$-hydroxysulfosuccinimide (sulfo-NHS) and $\mathrm{N}$-(3-(dimethylamino)propyl)$\mathrm{N}$-ethylcarbodiimide hydrochloride (EDC) ${ }^{26}$ (see also Supporting Information Section 2c). SA modified with $\mathrm{Cy} 3$ was employed to enable the simple verification of SA conjugation. After washing the product with phosphate-buffered saline (PBS), the fluorescence microscopy of SA-Cy3-modified $\mathrm{ZnMOF}-\mathrm{COOH}$ was obtained under laser irradiation at 543 $\mathrm{nm}$ (Figure S5). It was clear that the immobilization of SA-Cy3 on the composite surface had been successful.

It proved possible to manipulate $\mathrm{ZnMOF}-\mathrm{COOH}$ composite growth by modulating hydrothermal reaction time. After $24 \mathrm{~h}$ of heating, spherical particles $(\sim 5 \mu \mathrm{m}$ in diameter $)$ were generated (Figure 1a). However, extending reaction time gave larger particles with roughened surfaces (Figure $1 \mathrm{~b}-\mathrm{d}$ ). Finally, a reaction time of $120 \mathrm{~h}$ led to the generation of spherical particles $(\sim 150 \mu \mathrm{m}$ in diameter $)$ coated with spine-like structures (Figure 1e,f). It is established that the stiffness, charge, and superhydrophilicity ${ }^{26}$ as well as topology, roughness, and three-dimensional structure ${ }^{27}$ of a material influence its capacity for intercellular interaction and cell capture. In our case, detailed information was obtained by transmission electron microscopy (TEM, Figure S6). Results suggested the alignment of small needles (ca. $20 \mathrm{~nm}$ diameter) to form nanostructured agglomerates. The formation of these is consistent with the observation by SEM that ZnMOF-COOH displays a spine-like microstructure.

The grafting of biotinylated anti-EpCAM onto the composite following its modification with SA provides a multistep pathway to an immunotrapper capable of sequestering specific types of tumor cells. This work used MCF-7 as a model cell line (see
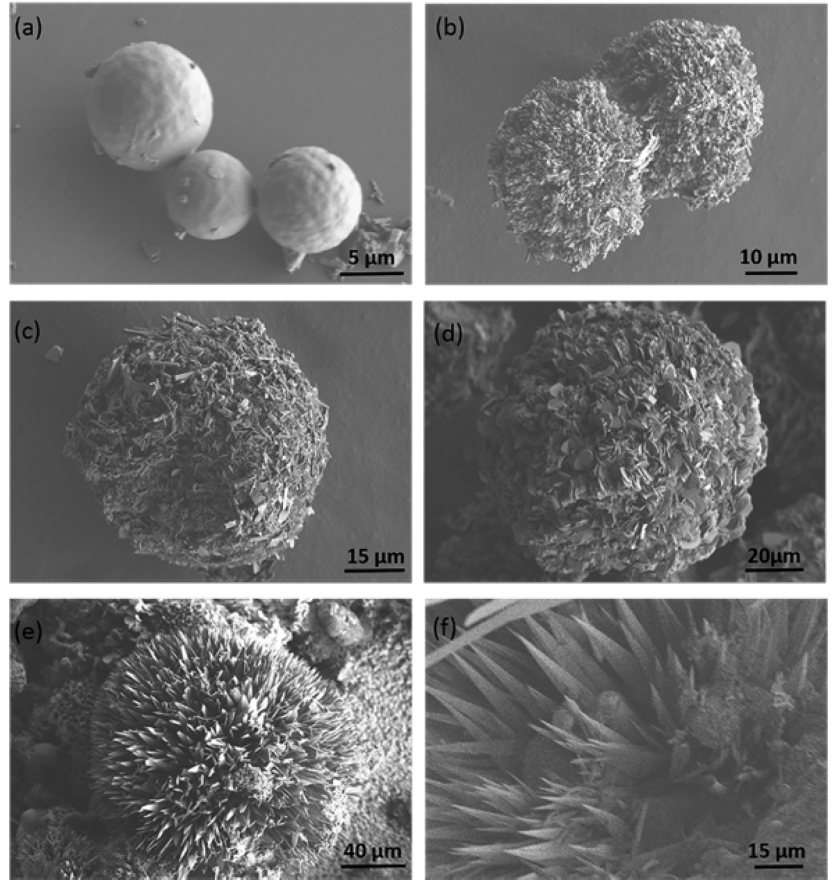

Figure 1. SEM images of $\mathrm{ZnMOF}-\mathrm{COOH}$ growing on $\mathrm{ZnO}$ substrate. Hydrothermal reaction times: (a) 24 h, (b) 48 h, (c) 72 h, (d) 96 h, and (e, f) $120 \mathrm{~h}$. Scale bars: (a) $5 \mu \mathrm{m}$, (b) $10 \mu \mathrm{m}$, (c) $15 \mu \mathrm{m}$, (d) 20 $\mu \mathrm{m}$, (e) $40 \mu \mathrm{m}$, and (f) $15 \mu \mathrm{m}$.

Supporting Information Section 3). Hence, a $1 \mathrm{~mL}$ MCF-7 suspension $\left(\sim 10^{4}\right.$ cells $\left./ \mathrm{mL}\right)$ was incubated with the immunotrapper at $37^{\circ} \mathrm{C}$ for $45 \mathrm{~min}$ in a $\mathrm{CO}_{2}$ incubator. Environmental scanning electron microscopy (ESEM) showed that MCF-7 cells were successfully captured, with the spines expressed by ZnMOF-COOH after PSM providing sites for cell sequestering (Figure $2 b-d$ ). To probe MCF-7 capture efficiency as a function of time, cells $(100$ cells $/ \mathrm{mL})$ were incubated with the trapping platform for 15-60 min (experiments were triplicated, $n=3$ ). Figure $2 \mathrm{e}$ reveals that capture efficiency increased from $64 \% \pm 11 \%$ at $15 \mathrm{~min}$ to $89 \% \pm 7 \%$ at $30 \mathrm{~min}$, only slightly increasing thereafter. As a result, $45 \mathrm{~min}$ was chosen as optimal incubation time, with capture efficiency of $91 \% \pm 5 \%$ achieved. The composite ZnMOF-COOH (without antibody conjugation) was monitored as a control. Incubation for $45 \mathrm{~min}$ revealed $96 \% \pm 1 \%$ MCF-7 cells remaining uncaptured (Figure $\left.2 \mathrm{e}, 45 \mathrm{~min}^{*}\right)$, demonstrating a key role for anti-EpCAM in cell recognition and capture.

Capture efficiency was studied as a function of immunotrapper morphology by conducting $45 \mathrm{~min}$ incubations using capture systems in which the ZnMOF-COOH components were fabricated over $24-120 \mathrm{~h}$ (Figure $2 \mathrm{f}, n=3$ ). Data revealed the morphology of the $\mathrm{ZnMOF}$ particles has a significant effect on capture efficiency. The relatively smooth, spherical particles produced after $24 \mathrm{~h}$ showed unsatisfactory capture efficiency (ca. 50\%). However, this rose to $>90 \%$ for the larger, less smooth particles that resulted from the $120 \mathrm{~h}$ synthesis. This indicated that, in accordance with previous results, ${ }^{26}$ a rougher biointerface benefits interaction of the targeted cells with the immunotrapper.

The low abundance of CTCs in the bloodstream made it desirable to test immunotrapper efficiency as a function of CTC concentration, with the levels tested varying from 10 cells to 100 cells $/ \mathrm{mL}$ in PBS. Figure $2 \mathrm{~g}(n=3)$ reveals that after 


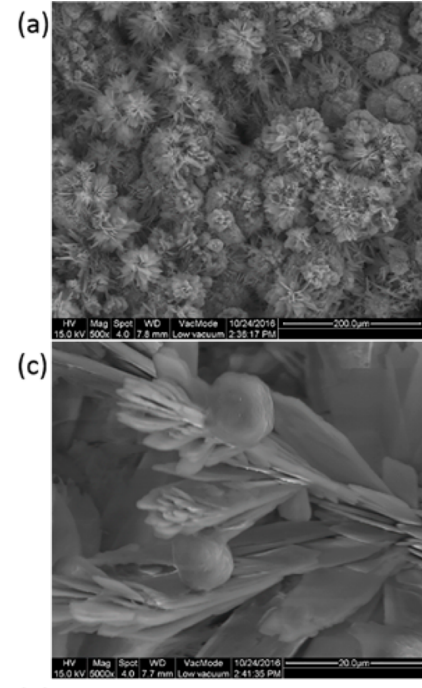

(e)

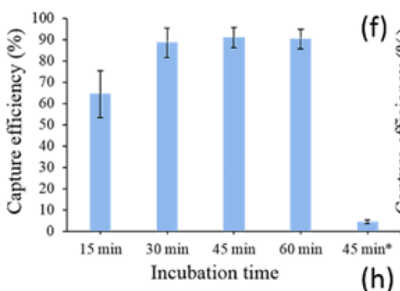

(h)
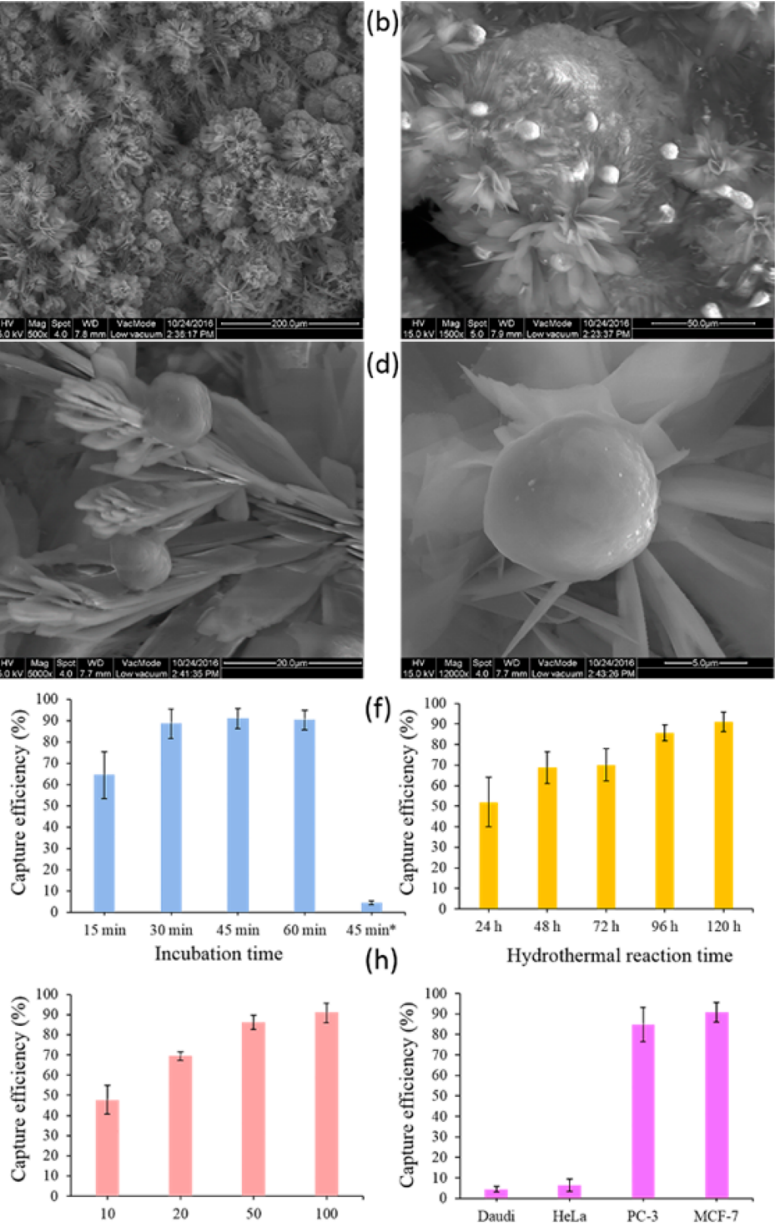

Cell numbers in $1 \mathrm{~mL}$ PBS

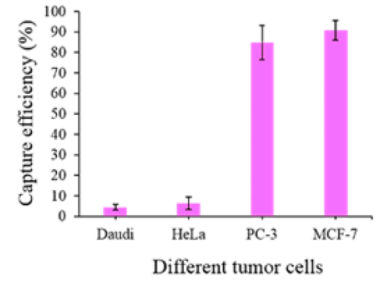

Figure 2. ESEM images of MCF-7 cells captured by a ZnMOF-based platform. (a) Overall morphology of ZnMOF-COOH after PSM; (bd) captured MCF-7 cells. Scale bars: (a) $200 \mu \mathrm{m}$, (b) $50 \mu \mathrm{m}$, (c) 20 $\mu \mathrm{m}$, (d) $5 \mu \mathrm{m}$. Immunotrapper capture efficiency (mean $\pm \mathrm{SD}, n=3$ ) as a function of (e) incubation time ( $45 \mathrm{~min}^{*}=45 \mathrm{~min}$ incubation using ZnMOF-COOH without anti-EpCAM conjugation), (f) hydrothermal preparation time of ZnMOF-COOH $(24-120 \mathrm{~h})$, (g) CTC concentration, and $(\mathrm{h})$ cell line.

incubation for $45 \mathrm{~min}$, capture efficiencies decreased from $91 \%$ $\pm 5 \%$ in $1 \mathrm{~mL}$ suspensions of $100 \mathrm{CTCs} / \mathrm{mL}$ to slightly lower than $50 \%$ for $10 \mathrm{CTCs} / \mathrm{mL}$. Our immunotrapper system was designed to target EpCAM-positive tumor cells. Different EpCAM-positive and EpCAM-negative cell lines were therefore used to verify discrimination. Human breast cancer cells (MCF7) and human prostate cancer cells (PC3) are EpCAM-positive, whereas human cervical cancer cells (HeLa) and Daudi lymphoma cells are EpCAM-negative. Figure $2 \mathrm{~h}(n=3)$ reveals a capture efficiency of PC-3 cells similar to that of MCF7 cells, reaching $85 \% \pm 8 \%$. In contrast, the figures for HeLa and Daudi cells were only $\sim 5 \%$. Overall, data in Figure 2 establish that the ZnMOF-based platform exhibits both excellent and targeted recognition of EpCAM-positive tumor cells.

To further elucidate cell adhesion on the surface of the ZnMOF-based immunotrapper, immunofluorescence analysis was undertaken. Representative laser confocal fluorescence microscopy images are shown in Figure 3 and Supporting Information Figure S7. MCF-7 cells were stained for the nuclei and actin filaments with 4',6-diamidino-2-phenylindole dihy-

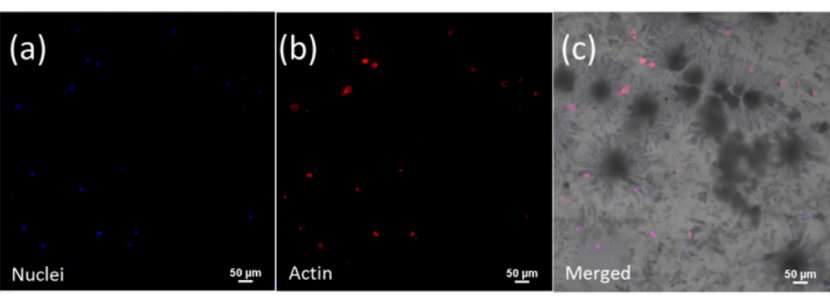

Figure 3. Immunofluorescent staining images showing captured MCF7 cells (100 cells $/ \mathrm{mL}, 45 \mathrm{~min}$ ). (a) Nuclei (DAPI, blue); (b) actin cytoskeletons (red); (c) composite image based on merged fluorescence images and bright field image of ZnMOF-based immunotrapper. Scale bars: $50 \mu \mathrm{m}$.

drochloride (DAPI, blue) and goat antirabbit IgG H\&L (Alexa Fluor 647) (red), respectively. Finally, the fluorescence images were merged with the corresponding bright field image of the immunotrapper. Figure 3 shows the distribution of actin cytoskeleton, a critically significant component in the interaction of tumor cells and immunotrapping material. ${ }^{28,29}$ The ESEM images shown in Figure $2 \mathrm{~b}-\mathrm{d}$ (see also Supporting Information Figure S8) as well as immunofluorescence analyses shown in Figures 3c and S7 all suggest the tumor cells show an affinity for regions in which the immunotrapper expresses a spine-like morphology. Taken together, these data suggest that in contrast to the core of the composite, which was formed in the early phase of the hydrothermal synthesis, the spine-like microstructures formed over longer timeframes provide sites at which cell adhesion is preferred and which, combined with the action of anti-EpCAM, enhance selective CTC capture efficiency.

Various functional materials have been developed for capturing tumor cells. While some have focused on optimal capture efficiency, ${ }^{26,27,30}$ others have focused on enhanced cell release after capture. ${ }^{29,31}$ However, while capture efficiencies greater than those we report have been recorded, ${ }^{26,32}$ there are, so far as we are aware, no reports of a multifunctional material capable of marrying efficient cell capture to drug delivery and controlled release. In light of this, we considered that beyond acting merely as a scaffold for a cell capture device, ZnMOF$\mathrm{COOH}$ could be deployed to host and release a therapeutic cargo in response to an external stimulus. ${ }^{19,33}$ Doxorubicin $(\text { Dox })^{4}$ was chosen as a model drug to test this idea (for detailed loading/release protocols see Supporting Information). Data in Figure S9 show that ZnMOF-COOH can load Dox easily at $\mathrm{pH}$ 7.4, reaching adsorption equilibrium (ca. 45\%) in only ca. 5 min. The loaded MOF showed $\mathrm{pH}$-sensitive Dox release in weakly acidic environments as shown in Figure S10. After incubation for $1 \mathrm{~h}$ in acid buffers ( $\mathrm{pH}$ 4.4-5.6), Dox desorption reached 50-55\%.

As a precursor to future bioapplications development, the cytotoxicity of the ZnMOF-COOH-based platform has been investigated. Live/Dead staining (Figure S11) showed 95\% \pm $1 \%$ cells to be viable after incubation for $45 \mathrm{~min}$, with statistically unchanged viability noted after $5 \mathrm{~h}$ of incubation. This compared with the viability of control groups remaining at ca. $96 \%$ over 5 h. Finally, cell viability in the presence of the platform decreased to $90 \% \pm 2 \%$ after incubation for $24 \mathrm{~h}$. These data indicated that CTC sequestering experiments should last $<24 \mathrm{~h}$ and that the current protocol for testing capture efficiency (see above, Figure 2e) operates on a time scale on which any cytotoxic effects of the platform manifest themselves negligibly. 
In conclusion, a functional MOF-based platform modified with antibodies has been developed to achieve cell recognition and targeted capture. ZnMOF-COOH assembled in situ on the surface of a $\mathrm{ZnO}$ substrate forms a convenient platform for further functionalization. This method offers a universal strategy for the immobilization of antibodies on MOFs by utilizing the straightforward derivatization of surface acid groups to give peptide linkages. The resulting ZnMOF-based platform acted as an efficient trapper of targeted tumor cells, exhibiting excellent capture capability and selectivity. Crucially, however, its structural properties also rendered the MOF capable of delivering drug cargoes and controllably releasing them in response to external stimuli. ${ }^{33}$ Overall, the development of a MOF-based immunotrapper system that successfully combines the fields of targeted cell sequestration with controllable drug delivery has enormous potential in personalized therapy development.

\section{ASSOCIATED CONTENT}

\section{S Supporting Information}

The Supporting Information is available free of charge on the ACS Publications website at DOI: 10.1021/acs.chemmater.7b03269.

Synthesis and materials characterization, fluorescence imaging, drug release data, and cytotoxicity tests (PDF) Fluorescence imaging (AVI)

\section{AUTHOR INFORMATION}

\section{Corresponding Authors}

*Andrew E. H. Wheatley. E-mail: aehw2@cam.ac.uk.

*Huwei Liu. E-mail: hwliu@pku.edu.cn.

\section{ORCID $\odot$}

Yu Bai: 0000-0003-1542-0297

Andrew E. H. Wheatley: 0000-0002-2624-6063

Huwei Liu: 0000-0001-6540-9657

\section{Author Contributions}

All authors have given their approval to the final version of the manuscript.

\section{Funding}

This work was financially supported by the National Natural Science Foundation of China (Grant Nos. 21527809 and 21575007), the China Scholarship Council and the UK EPSRC (EP/J500380/1).

Notes

The authors declare no competing financial interest.

\section{REFERENCES}

(1) Silva, P.; Vilela, S. M.; Tome, J. P.; Almeida Paz, F. A. A. Multifunctional Metal-Organic Frameworks: From Academia to Industrial Applications. Chem. Soc. Rev. 2015, 44, 6774-6803.

(2) Giménez-Marqués, M.; Hidalgo, T.; Serre, C.; Horcajada, P. Nanostructured Metal-Organic Frameworks and Their Bio-Related Applications. Coord. Chem. Rev. 2016, 307, 342-360.

(3) He, C.; Liu, D.; Lin, W. Nanomedicine Applications of Hybrid Nanomaterials Built from Metal-Ligand Coordination Bonds: Nanoscale Metal-Organic Frameworks and Nanoscale Coordination Polymers. Chem. Rev. 2015, 115, 11079-11108.

(4) Zheng, H.; Zhang, Y.; Liu, L.; Wan, W.; Guo, P.; Nystrom, A. M.; Zou, X. One-pot Synthesis of Metal-Organic Frameworks with Encapsulated Target Molecules and Their Applications for Controlled Drug Delivery. J. Am. Chem. Soc. 2016, 138, 962-968.
(5) Levine, D. J.; Runcevski, T.; Kapelewski, M. T.; Keitz, B. K.; Oktawiec, J.; Reed, D. A.; Mason, J. A.; Jiang, H. Z.; Colwell, K. A.; Legendre, C. M.; FitzGerald, S. A.; Long, J. R. Olsalazine-Based MetalOrganic Frameworks as Biocompatible Platforms for $\mathrm{H}_{2}$ Adsorption and Drug Delivery. J. Am. Chem. Soc. 2016, 138, 10143-10150.

(6) Tellez-Gabriel, M.; Ory, B.; Lamoureux, F.; Heymann, M.-F.; Heymann, D. Tumour Heterogeneity: The Key Advantages of SingleCell Analysis. Int. J. Mol. Sci. 2016, 17, 2142.

(7) Jackson, J. M.; Witek, M. A.; Kamande, J. W.; Soper, S. A. Materials and Microfluidics: Enabling the Efficient Isolation and Analysis of Circulating Tumour Cells. Chem. Soc. Rev. 2017, 46, 42454280.

(8) Kaiser, J. Cancer's Circulation Problem. Science 2010, 327, 10721074.

(9) Tang, M.; Wen, C. Y.; Wu, L. L.; Hong, S. L.; Hu, J.; Xu, C. M.; Pang, D. W.; Zhang, Z. L. A Chip Assisted Immunomagnetic Separation System for the Efficient Capture and in situ Dentification of Circulating Tumor Cells. Lab Chip 2016, 16, 1214-1223.

(10) Steeg, P. S. Tumor Metastasis: Mechanistic Insights and Clinical Challenges. Nat. Med. 2006, 12, 895-904.

(11) Kwak, B.; Lee, J.; Lee, D.; Lee, K.; Kwon, O.; Kang, S.; Kim, Y. Selective Isolation of Magnetic Nanoparticle-Mediated Heterogeneity Subpopulation of Circulating Tumor Cells Using Magnetic Gradient Based Microfluidic System. Biosens. Bioelectron. 2017, 88, 153-158.

(12) Sarioglu, A. F.; Aceto, N.; Kojic, N.; Donaldson, M. C.; Zeinali, M.; Hamza, B.; Engstrom, A.; Zhu, H.; Sundaresan, T. K.; Miyamoto, D. T.; Luo, X.; Bardia, A.; Wittner, B. S.; Ramaswamy, S.; Shioda, T.; Ting, D. T.; Stott, S. L.; Kapur, R.; Maheswaran, S.; Haber, D. A.; Toner, M. A Microfluidic Device for Label-Free, Physical Capture of Circulating Tumor Cell Clusters. Nat. Methods 2015, 12, 685-691.

(13) Liu, H.; Li, Y.; Sun, K.; Fan, J.; Zhang, P.; Meng, J.; Wang, S.; Jiang, L. Dual-Responsive Surfaces Modified with Phenylboronic AcidContaining Polymer Brush to Reversibly Capture and Release Cancer Cells. J. Am. Chem. Soc. 2013, 135, 7603-7609.

(14) Chen, L.; Ye, J.-W.; Wang, H.-P.; Pan, M.; Yin, S.-Y.; Wei, Z.W.; Zhang, L.-Y.; Wu, K.; Fan, Y.-N.; Su, C.-Y. Ultrafast Water Sensing and Thermal Imaging by a Metal-Organic Framework with Switchable Luminescence. Nat. Commun. 2017, 8, 15985.

(15) Yang, Q.; Xu, Q.; Yu, S.-H.; Jiang, H.-L. Pd Nanocubes@ZIF-8: Integration of Plasmon-Driven Photothermal Conversion with a Metal-Organic Framework for Efficient and Selective Catalysis. Angew. Chem., Int. Ed. 2016, 55, 3685-3689.

(16) Osta, W. A.; Chen, Y.; Mikhitarian, K.; Mitas, M.; Salem, M.; Hannun, Y. A.; Cole, D. J.; Gillanders, W. E. EpCAM is Overexpressed in Breast Cancer and is a Potential Target for Breast Cancer Gene Therapy. Cancer Res. 2004, 64, 5818-5824.

(17) Cao, J.; Gao, Y.; Wang, Y.; Du, C.; Liu, Z. A Microporous MetalOrganic Open Framework Containing Uncoordinated Carbonyl Groups as Postsynthetic Modification Sites for Cation Exchange and $\mathrm{Tb}^{3+}$ Sensing. Chem. Commun. 2013, 49, 6897-6899.

(18) Gadzikwa, T.; Farha, O. K.; Mulfort, K. L.; Hupp, J. T.; Nguyen, S. T. A Zn-Based, Pillared Paddlewheel MOF Containing Free Carboxylic Acids via Covalent Post-Synthesis Elaboration. Chem. Commun. 2009, 3720-3722.

(19) Liu, Y.; Xi, X.; Ye, C.; Gong, T.; Yang, Z.; Cui, Y. Chiral Metalorganic Frameworks Bearing Free Carboxylic Acids for Organocatalyst Encapsulation. Angew. Chem., Int. Ed. 2014, 53, 13821-13825.

(20) Karagiaridi, O.; Vermeulen, N. A.; Klet, R. C.; Wang, T. C.; Moghadam, P. Z.; Al-Juaid, S. S.; Stoddart, J. F.; Hupp, J. T.; Farha, O. K. Functionalized Defects Through Solvent-Assisted Linker Exchange: Synthesis, Characterization, and Partial Postsynthesis Elaboration of a Metal-Organic Framework Containing Free Carboxylic Acid Moieties. Inorg. Chem. 2015, 54, 1785-1790.

(21) Kumar, P.; Kim, K.-H.; Bansal, V.; Paul, A. K.; Deep, A. Practical Utilization of Nanocrystal Metal Organic Framework Biosensor for Parathion Specific Recognition. Microchem. J. 2016, 128, 102-107.

(22) Kumar, P.; Kumar, P.; Bharadwaj, L. M.; Paul, A. K.; Deep, A. Luminescent Nanocrystal Metal Organic Framework Based Biosensor for Molecular Recognition. Inorg. Chem. Commun. 2014, 43, 114-117. 
(23) Pivetal, J.; Pereira, F. M.; Barbosa, A. I.; Castanheira, A. P.; Reis, N. M.; Edwards, A. D. Covalent Immobilisation of Antibodies in Teflon-FEP Microfluidic Devices for the Sensitive Quantification of Clinically Relevant Protein Biomarkers. Analyst 2017, 142, 959-968.

(24) Wiseman, M. E.; Frank, C. W. Antibody Adsorption and Orientation on Hydrophobic Surfaces. Langmuir 2012, 28, 17651774.

(25) Atacan, K.; Çakıroğlu, B.; Özacar, M. Covalent Immobilization of Trypsin onto Modified Magnetite Nanoparticles and its Application for Casein Digestion. Int. J. Biol. Macromol. 2017, 97, 148-155.

(26) Li, Y.; Lu, Q.; Liu, H.; Wang, J.; Zhang, P.; Liang, H.; Jiang, L.; Wang, S. Antibody-Modified Reduced Graphene Oxide Films with Extreme Sensitivity to Circulating Tumor Cells. Adv. Mater. 2015, 27, 6848-6854.

(27) Liu, X.; Chen, L.; Liu, H.; Yang, G.; Zhang, P.; Han, D.; Wang, S.; Jiang, L. Bio-Inspired Soft Polystyrene Nanotube Substrate for Rapid and Highly Efficient Breast Cancer-Cell Capture. NPG Asia Mater. 2013, 5, e63.

(28) Zhang, F.; Jiang, Y.; Liu, X.; Meng, J.; Zhang, P.; Liu, H.; Yang, G.; Li, G.; Jiang, L.; Wan, L.-J.; Hu, J.-S.; Wang, S. Hierarchical Nanowire Arrays as Three-Dimensional Fractal Nanobiointerfaces for High Efficient Capture of Cancer Cells. Nano Lett. 2016, 16, 766-772.

(29) Ouyang, J.; Chen, M.; Bao, W.-J.; Zhang, Q.-W.; Wang, K.; Xia, X.-H. Morphology Controlled Poly(aminophenylboronic Acid) Nanostructures as Smart Substrates for Enhanced Capture and Release of Circulating Tumor Cells. Adv. Funct. Mater. 2015, 25, 6122-6130.

(30) Zheng, F.; Cheng, Y.; Wang, J.; Lu, J.; Zhang, B.; Zhao, Y.; Gu, Z. Aptamer-Functionalized Barcode Particles for the Capture and Detection of Multiple Types of Circulating Tumor Cells. Adv. Mater. 2014, 26, 7333-7338.

(31) Reategui, E.; Aceto, N.; Lim, E. J.; Sullivan, J. P.; Jensen, A. E.; Zeinali, M.; Martel, J. M.; Aranyosi, A. J.; Li, W.; Castleberry, S.; Bardia, A.; Sequist, L. V.; Haber, D. A.; Maheswaran, S.; Hammond, P. T.; Toner, M.; Stott, S. L. Tunable Nanostructured Coating for the Capture and Selective Release of Viable Circulating Tumor Cells. Adv. Mater. 2015, 27, 1593-1599.

(32) Yoon, H. J.; Kim, T. H.; Zhang, Z.; Azizi, E.; Pham, T. M.; Paoletti, C.; Lin, J.; Ramnath, N.; Wicha, M. S.; Hayes, D. F.; Simeone, D. M.; Nagrath, S. Sensitive Capture of Circulating Tumour Cells by Functionalized Graphene Oxide Nanosheets. Nat. Nanotechnol. 2013, $8,735-741$.

(33) Song, J. Y.; Ahmed, I.; Seo, P. W.; Jhung, S. H. UiO-66-type Metal-organic Framework with Free Carboxylic Acid: Versatile Adsorbents via $\mathrm{H}$-Bond for Both Aqueous and Nonaqueous Phases. ACS Appl. Mater. Interfaces 2016, 8, 27394-27402. 